\title{
Design of an everting balloon to deploy a microendoscope to the fallopian tubes.
}

\author{
Molly Keenan ${ }^{1}$, Caitlin Howard ${ }^{1}$, Tyler Tate ${ }^{2,}$ Ian McGuiness $^{3}$, Alexis Sauer-Budge ${ }^{3,4}$, John Black ${ }^{5,}$ Urs Utzinger $^{1,2}$, \\ Jennifer K Barton ${ }^{1,2}$ \\ ${ }^{1}$ Biomedical Engineering, University of Arizona, Tucson, $A Z^{2}$ College of Optical Sciences, University of Arizona, \\ Tucson, AZ ${ }^{3}$ Fraunhofer USA - CMI ,Boston, MA ${ }^{4}$ Biomedical Engineering, Boston University, MA ${ }^{5}$ Glannaventa, Inc.
}

\begin{abstract}
The 5-year survival rate for ovarian cancer is only $45 \%$ largely due to lack of effective screening methods. Current methods include palpation, transvaginal ultrasound, and the CA-125 blood test. Finding disease reliably and at an early stage increase survival to $92 \%$. We have designed and built a $0.7 \mathrm{~mm}$ endoscope for the early detection of ovarian cancer. Inserted transvaginally through the working channel of a hysteroscope, the falloposcope creates a minimally invasive procedure for the screening of high risk women. To improve the ease-of-use and safety of falloposcope deployment, we are working to create an everting balloon. Currently, the falloposcope would require a skilled user to operate due to the challenging anatomy of the fallopian tubes - a small opening from the uterus $(<1 \mathrm{~mm})$, tortuous path, and delicate lumenal features. A balloon delivery system would gently open the fallopian tube and guide the falloposcope down the center of lumen. We show balloon design and discuss integration with the falloposcope prototype. We test possible mechanical damage to the tissue due to scraping, puncture, or overstretching. Successful introduction of the everting balloon to simplify falloposcope delivery could expand screening beyond specialized centers to smaller clinical locations.
\end{abstract}

Keywords: burst testing, safety, efficacy, pressure, introducer sheath, screening test

\section{INTRODUCTION}

Recent research suggests that the fallopian tubes play an important role in the origin of ovarian cancer, making it a critical tissue to image for early stage disease. [1] Imaging of the fallopian tubes was performed in the early 1990s to investigate the cause of infertility. [2], [3] These methods included direct cannulation of the fallopian tube, guide-wire directed cannulation, and a linear everting balloon catheter that could open the tube when closures were found. Early falloposcopes were $0.5 \mathrm{~mm}$ in diameter using simple white light and 6,000 element fiber bundles. [4] Falloposcopy was successful with eighty falloposcopic procedures performed in vivo and a scoring system developed for salpingitis. [5] These early falloposcopes, while small enough to pass through the full length, were sensitive enough only to see blockages of the tube.

More sensitive systems are required to study and detect the as-of-yet unknown changes that occur in the beginning of cancer. Our aim is to gain information of high-grade serous ovarian cancer, the form responsible for almost $90 \%$ of death from ovarian cancer, at a cellular level. [6] Recent attempts have been made at interrogating the fallopian tube for this purpose. A recent study to gather dysplastic cells was performed on the fallopian tubes of women undergoing laparoscopic hysterectomy. [7] This study used a $3.2 \mathrm{Fr}(1 \mathrm{~mm})$ brush for hysteroscopic cytology and a $5 \mathrm{Fr}$ brush for laparoscopic sampling, but could only reach approximately $3 \mathrm{~cm}$ into the proximal end. [8] Perhaps the first attempt to image the lumen of the fallopian tube from a proximal introduction for cancer in vivo was performed with a forwardviewing scanning fiber endoscope. [9] This is a 3.6 Fr $(1.2 \mathrm{~mm})$ imaging probe introduced through the ostia with the aid of a $5 \operatorname{Fr}(1.7 \mathrm{~mm})$ cannula and saline flush. [9] Difficulty passing through the first portions of the fallopian tube were also reported. Due to this difficulty, others have tried a laparoscopic approach, to enter from the distal end of the fallopian tubes [10]

Photonic Therapeutics and Diagnostics XII, edited by Bernard Choi, et al., Proc. of SPIE Vol. 9689, $968944 \cdot$ C C 2016 SPIE · CCC code: 1605-7422/16/\$18 · doi: 10.1117/12.2213345 
These studies suggest that a smaller endoscope, under $1 \mathrm{~mm}$, with an aid in opening the fallopian tube, are required. Falloposcopy was performed successfully in the 1990s to investigate causes of infertility. [11] The study found that the use of a linear everting balloon is helpful to get past strictures and delicate plicae that would otherwise stop a guide wire. Another benefit of a balloon introducer is its ability to center the falloposcope in the lumen, which aids in optimizing the working distance for the imaging system.

In order to optimize the design and use of a balloon as an introducer and the optimal size of a falloposcope, we need to better understand the anatomy and mechanical behavior of the fallopian tube. Gross fallopian tube anatomy is well known. In humans, the fallopian tube is approximately $10-12 \mathrm{~cm}$ long, connecting the ovaries and uterus. The lumen is filled with epithelium covered fronds, plicae, surrounded by circular smooth muscle of varying thickness and interstitial connective tissue. The ostia, the opening to the fallopian tube from the uterus, is approximately $1 \mathrm{~mm}$ in diameter. The tube itself can be divided into three segments (ordered proximal to distal), the isthmus, ampulla and infundibulum, increasing in lumen diameter to up to $1 \mathrm{~cm}$. The infundibulum ends in fimbriae, the finger-like projections that brush the ovary surface. While the uterus changes dramatically between humans and pigs, porcine fallopian tubes are remarkably similar to humans, and are more easily available - making them a good model system in which to test our prototype falloposcope. Minor differences are that porcine tubes tubes are longer and can be more tortuous. [12], [13]

This proceedings paper covers the results of the preliminary pressure testing on porcine fallopian tubes. The first goal is to understand the normal fallopian tube microscopic anatomy. Once we understand this, we can study the effect of pressure and balloon material interaction on the lumen of the fallopian tube to determine the levels of pressurization that are safe and efficacious for linear everting balloon deployment. We link these results to the design of the everting balloon and discuss future directions.

\section{EVERTING BALLOON}

To improve ease of access to the fallopian tube, we are developing a linear everting balloon, shown in figure 1 . This balloon is connected to two telescoping tubes, and inner tube and an outer tube. The space between these tubes is filled with saline. Once pressurized with saline, the balloon expands into the fallopian tube and pulls the inner tube inside as well. The falloposcope can be introduced through this channel. This has an advantage over guidewire -based cannulation because it pushes the fronds of the fallopian tubes out of the way.
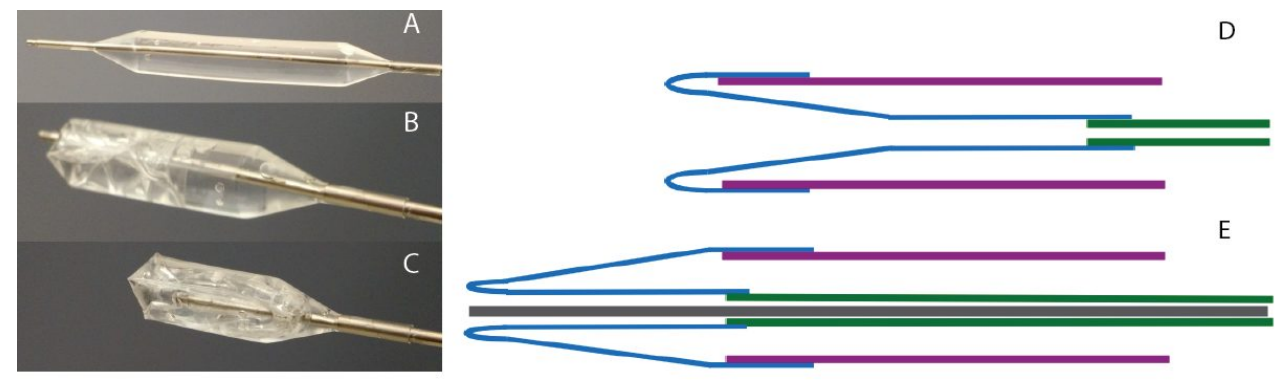

Figure 1-Testing of tapered balloon and pressurization system (right). A) Balloon under pressure. B) Balloon fully everted. C) Balloon in the beginning conformation.. Falloposcope schematic (right) showing the falloposcope (gray bar) in the lumen of the inner tube (green). The inner tube is connected to the outer tubing (purple) by the balloon (blue), shown here doubled over on itself.

The current prototype uses a low durometer urethane attached to two nested stainless steel hypotubes. The balloon wall thickness is $0.0254 \mathrm{~mm}$, diameter is $8 \mathrm{~mm}$ and is $40 \mathrm{~mm}$ long. This large diameter balloon is being used to test a prototype handle. The handle has two chambers separated by a sealed piston. Continuous with the inside of the balloon is saline and on the right channel is air. 

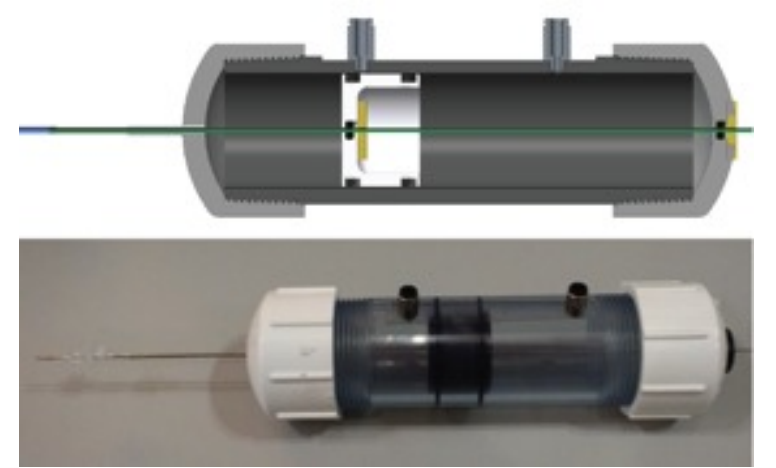

Figure 2 - Pressurization handle prototype as a Solidworks schematic (top) and physical prototype (bottom). This shows a 2-chamber design with a movable piston. The left side, continuous with the balloon, is filled with saline. The right side is pressurized air.

\section{MATERIALS AND METHODS}

\subsection{Specimens}

Five full porcine reproductive tracts were acquired from local meat processing plants. Reproductive tracts were kept cold on ice for up to 24 hours before testing. Prior to inflation, the fallopian tubes were dissected away from the uterus and removed from the connective tissue responsible for its tortuosity. The fallopian tubes were sectioned into three pieces, approximately six centimeters each. Distal segments included the transition to fimbriae, proximal segments included 1 $\mathrm{mm}$ of the uterine junction, and the middle was the segment connecting the other two. Experiments were performed at room temperature.

\section{2 $\quad$ Pressure testing}

Samples were inflated by a syringe with a threaded plunger and saline in order reach pressure greater than $3 \mathrm{~atm}$. The syringe has a Luer lock tip which attaches to disposable tubing. The fallopian tube is firmly attached to this tubing with with suture (shown in figure 3) or cyanoacrylate for the smallest diameter. Saline is flushed through the tube to remove air and the distal end is clamped shut with hemostats. Pressure is increased until the tube bursts in an area $1 \mathrm{~mm}$ away from attachment sites.

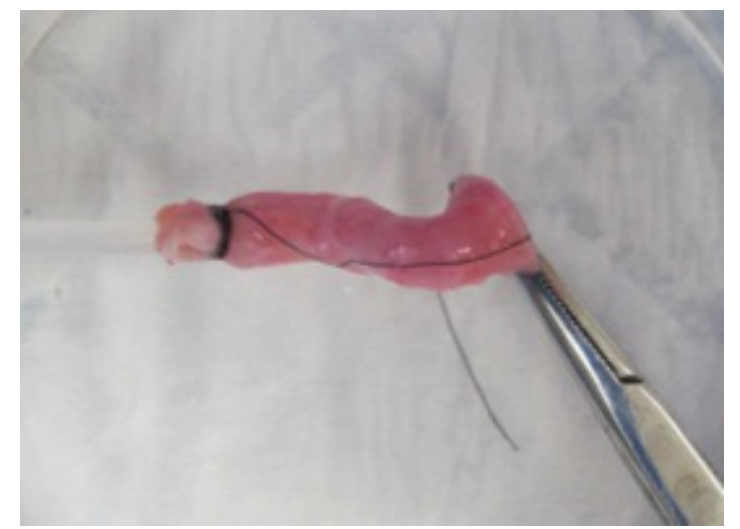

Figure 3 - Inflated ampulla portion of porcine fallopian tube. This shows the tube attached to the tubing with sutures and the other end clamped shut. The outer diameter does change when saline is introduced into the tube.

\subsection{Processing}

The burst portions were then cut into two $1 \mathrm{~mm}$ sections, removing the portion affected by our instruments, and fixed in $10 \%$ formalin. Fallopian tubes were embedded in paraffin and sectioned perpendicular to the lumen. We used the 
Masson's Trichrome stain (connective tissue is blue, epithelial and muscle tissue is red) in order to differentiate epithelium and muscle tissue from connective tissue. Images were analyzed qualitatively for epithelial disruption due to scraping. Measurements were taken of inner diameters, outer diameters, and wall thickness with Olympus MicroSuite software.

\section{$4 \quad$ RESULTS}

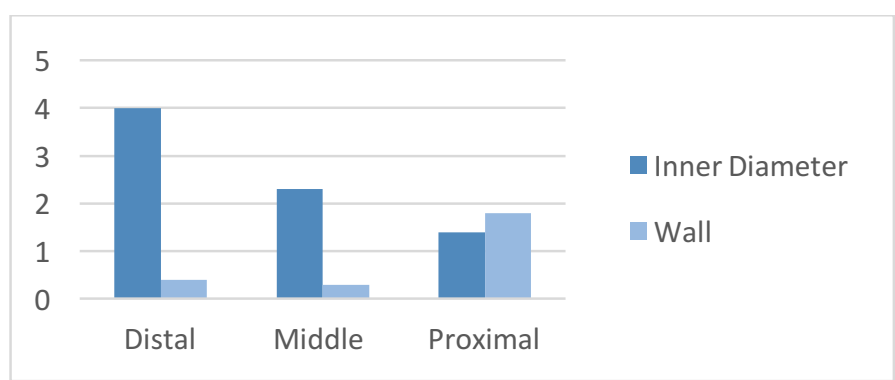

Figure 4 - Inner diameter and wall thickness measurements in millimeters.

Figure 4 shows the average measurements, in millimeters, for the inner diameter and the wall. We defined the inner diameter as the distance across the fronds and the wall as the thickness of the smooth muscle. Measurements reported were taken on fixed control tissue using Olympus MicroSuite software.

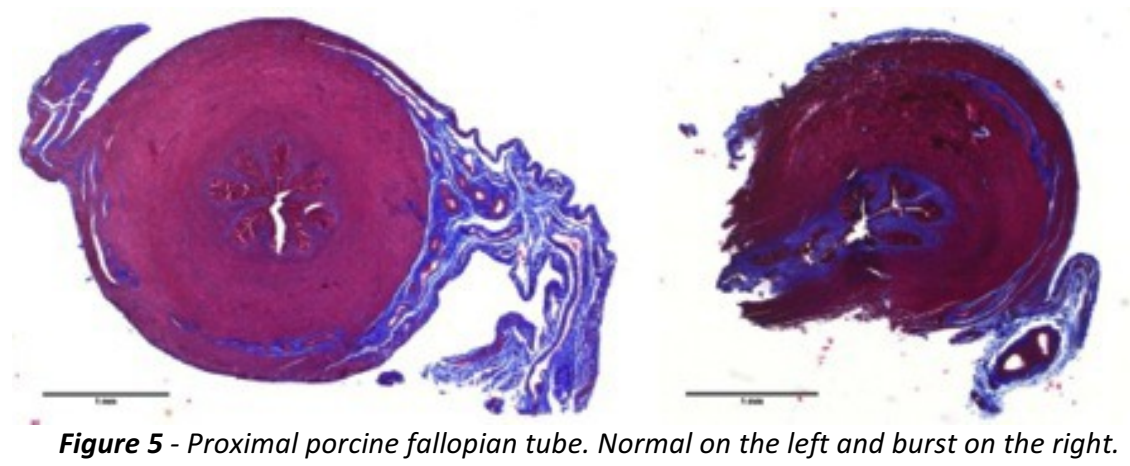

Proximal portions, figure 5, have the highest ratio of smooth muscle to inner lumen, even though the outer diameter is the smallest of the whole segment. The fallopian tube is attached to the body with connective tissue rich in blood vessels. This is shown in some sections and not others depending on how the dissection was performed. The burst tissue on the right shows a severe injury. Here, the outer connective tissue, the smooth muscle and the inner connective tissue have been ruptured. Proximal tissue bursts at significantly higher pressures than more distal portions do. 

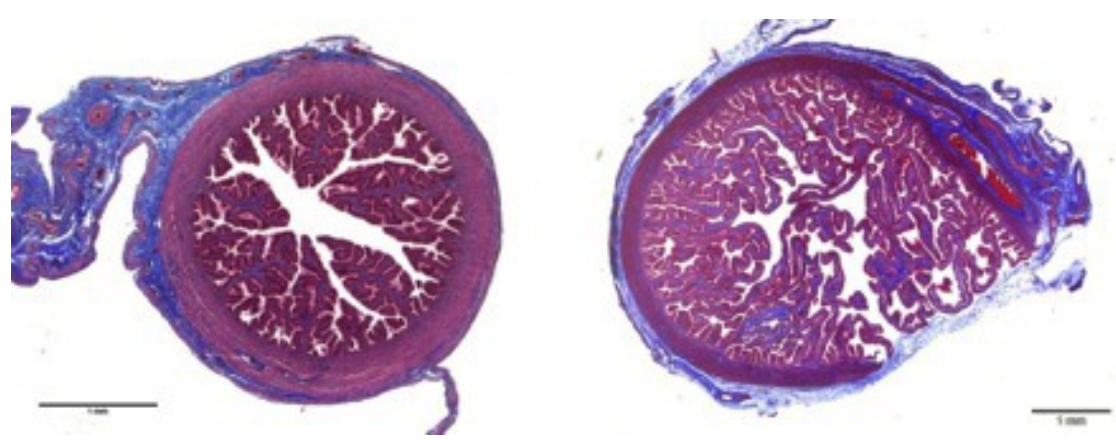

Figure 6 - Middle porcine fallopian tubes. Normal on the left and burst on the right.

The middle portion of the fallopian tube, shown in figure 6 , has a smaller ratio of muscle to lumen. The fronds still protrude into the tube but it is more common to see space, though this is affected by the fixation process. The burst portion shows another common injury. Here, the smooth muscle ruptures but the outer connective tissue is stretched and thinned, but has not fully broken.
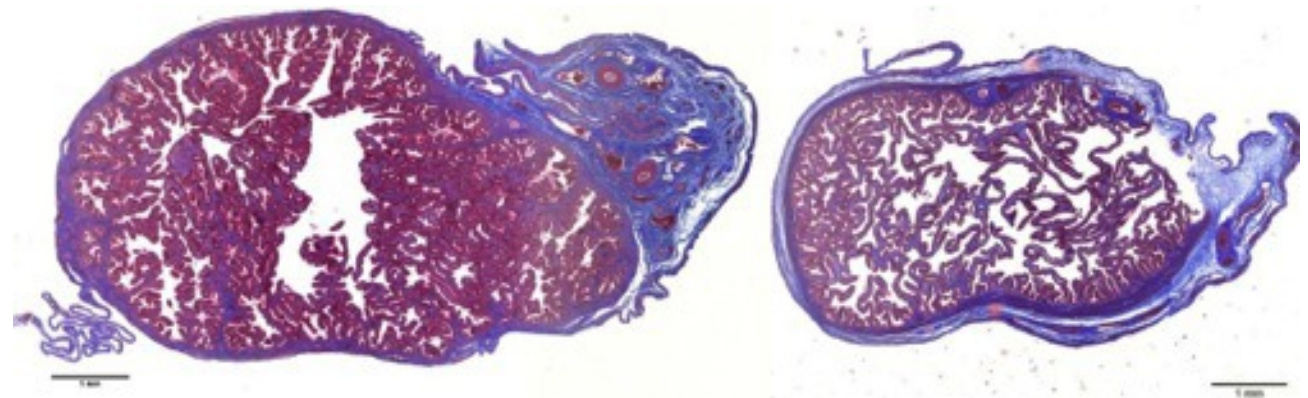

Figure 7 - Distal porcine fallopian tubes. Normal on the left and burst on the right.

The distal fallopian tube shows the greatest variability in size, as shown in figure 7 . The fronds are more delicate and the lumen is the largest. The smooth muscle almost disappears and connective tissue dominates. This is also the portion in the gross anatomy that has the greatest freedom to move around. The burst distal portion again shows that, while the inner portion becomes disconnected, that tube is held together by the outer connective tissue.

\section{DISCUSSION}

\subsection{Fallopian tube diameters}

We measured the diameters of the porcine fallopian tubes in order to more accurately compare to human tissue. Because the lengths of the fallopian tubes differ for pigs and humans, we aim to relate burst pressure in the animal model to human tissue through wall thickness. We are perfecting our method to acquire measurements pre- and post-testing to assess changes in diameter once inflated. Preliminary data show that wall thickness does not change in burst and control fallopian tubes. However, after saline is introduced, the outer diameter increases to some maximum diameter. After this pressure is reached, further increases cause the structure to fail abruptly, rather than stretching further.

\subsection{Burst testing}

In general, we noticed two failure modes: the first, the serosa stays intact and expands, and second, a hole is formed through smooth muscle and connective tissue. It is worth reiterating that this is the worst-case scenario. We do not intend to come near this bursting pressure, in this preliminary study approximately $3 \mathrm{~atm}$, in the fallopian tubes, and aim to inflate the balloon at a threshold below bursting. Further tests will be done to assess the appearance of early stage disruption/perforation at this lower pressure. 
The proximal portion contains the ostia, the opening to the uterus, and the narrowest portions of the fallopian tube. We do not anticipate bursting this part of the tissue due to its thick muscle walls, often greater than the diameter of the lumen itself. From our section by section testing, we note that the thicker muscle regions can withstand higher bursting pressures. In future tests we will assess if this area will stretch to accommodate the balloon and falloposcope.

The portion most at risk for bursting is the ampulla, the middle portion of the fallopian tube. This is the portion that has a relatively thin wall and narrow lumen. Common failures are tearing of the smooth muscle wall, with saline subsequently filling the space within the surrounding connective tissue. Failures were also seen at the distal end of the fallopian tubes leading up to the fimbriae, where the wall is the thinnest. However, we don't expect this portion to cause problems when the full tube is inflated because the ratio of smooth muscle thickness to lumen diameter places the ampulla at more risk.

\section{CONCLUSION}

We have performed preliminary tests to evaluate the mechanical behavior of porcine fallopian tubes. For future tests, we will collect data from a digital pressure sensor during inflation to accurately measure maximum bursting pressure. This result will determine the maximum inflation diameter of the balloon, which will effect pressurization mechanics and material choice. After delivery of the custom balloon, we will assess barotrauma as well as mechanical trauma to the epithelial lining. Finally, we will collect samples of human tissue to compare with the porcine model.

\section{REFERENCES}

[1] R. S. Tuma, "Origin of Ovarian Cancer May Have Implications for Screening," J. Natl. Cancer Inst., vol. 102, no. 1, pp. 11-13, Jan. 2010.

[2] G. N. Allahbadia and R. Merchant, "Fallopian tube recanalization: lessons learnt and future challenges," Womens Health Lond. Engl., vol. 6, no. 4, pp. 531-548, quiz 548-549, Jul. 2010.

[3] J. Kerin, E. Surrey, L. Daykhovsky, and W. S. Grundfest, "Development and application of a falloposcope for transvaginal endoscopy of the fallopian tube," J. Laparoendosc. Surg., vol. 1, no. 1, pp. 47-56, 1990.

[4] M. P. Milad and R. S. Corfman, "Falloposcopy," Curr. Opin. Obstet. Gynecol., vol. 4, no. 3, pp. 406-411, Jun. 1992.

[5] J. F. Kerin, E. S. Surrey, D. B. Williams, L. Daykhovsky, and W. S. Grundfest, "Falloposcopic Observations of Endotubal Isthmic Plugs as a Cause of Reversible Obstruction and Their Histological Characterization," $J$. Laparoendosc. Surg., vol. 1, no. 2, pp. 103-110, Jan. 1991.

[6] S. Vaughan, J. I. Coward, R. C. Bast, A. Berchuck, J. S. Berek, J. D. Brenton, G. Coukos, C. C. Crum, R. Drapkin, D. Etemadmoghadam, M. Friedlander, H. Gabra, S. B. Kaye, C. J. Lord, E. Lengyel, D. A. Levine, I. A. McNeish, U. Menon, G. B. Mills, K. P. Nephew, A. M. Oza, A. K. Sood, E. A. Stronach, H. Walczak, D. D. Bowtell, and F. R. Balkwill, "Rethinking ovarian cancer: recommendations for improving outcomes," Nat. Rev. Cancer, vol. 11, no. 10, pp. 719-725, Oct. 2011.

[7] D. Lum, R. Guido, E. Rodriguez, T. Lee, S. Mansuria, L. D'Ambrosio, and R. M. Austin, "Brush Cytology of the Fallopian Tube and Implications in Ovarian Cancer Screening," J. Minim. Invasive Gynecol., vol. 21, no. 5, pp. 851-856, Sep. 2014.

[8] E. F. Rodriguez, D. Lum, R. Guido, and R. M. Austin, "Cytologic Findings in Experimental in vivo Fallopian Tube Brush Specimens," Acta Cytol., vol. 57, no. 6, pp. 611-618, 2013.

[9] E. J. Seibel, C. D. Melville, R. S. Johnston, Y. Gong, K. Agnew, S. Chiang, and E. M. Swisher, "In vivo laser-based imaging of the human fallopian tube for future cancer detection," 2015, vol. 9304, p. 93040Q-93040Q-9.

[10] A. A. Tanbakuchi, A. R. Rouse, J. A. Udovich, K. D. Hatch, and A. F. Gmitro, "Clinical confocal microlaparoscope for real-time in vivo optical biopsies," J. Biomed. Opt., vol. 14, no. 4, p. 044030, 2009.

[11] S. B. Benjamin and K. Collins, "The toposcopic through-lumen everting catheter to facilitate dilation of severe strictures of the gastrointestinal tract," Gastrointest. Endosc.

[12] I. Briceag, A. Costache, V. Purcarea, R. Cergan, M. Dumitru, I. Briceag, M. Sajin, and A. Ispas, "Fallopian tubes literature review of anatomy and etiology in female infertility," J. Med. Life, vol. 8, no. 2, pp. 129-131, 2015.

[13] E. Lorenzen, F. Follmann, G. Jungersen, and J. S. Agerholm, "A review of the human vs. porcine female genital tract and associated immune system in the perspective of using minipigs as a model of human genital Chlamydia infection," Vet. Res., vol. 46, 2015. 